\title{
Ancestry-Informative Marker
}

National Cancer Institute

\section{Source}

National Cancer Institute. Ancestry-Informative Marker. NCI Thesaurus. Code C104680.

A set of genetic polymorphisms that are found in different frequencies between populations from different geographical regions. 